九州大学学術情報リポジトリ

Kyushu University Institutional Repository

\title{
Multi-physics Extension of OpenFMO Framework
}

Takami, Toshiya

Research Institute for Information Technology, Kyushu University

Maki, Jun

Research Institute for Information Technology, Kyushu University

Ooba, Junichi

Research Institute for Information Technology, Kyushu University

Inadomi, Yuichi

PSI Project Lab., Kyushu University

他

http://hdl. handle. net/2324/9181

出版情報: International Conference of Computational Method in Sciences and Enginnering. 2007, 2007-09-25

バージョン :

権利関係 : 


\title{
Multi-physics Extension of OpenFMO Framework
}

\author{
Toshiya Takami*, Jun Maki*, Jun'ichi Ooba*, Yuuichi Inadomi ${ }^{\dagger}$, Hiroaki Honda ${ }^{\dagger}$, \\ Ryutaro Susukita**, Koji Inoue ${ }^{\ddagger \dagger}$, Taizo Kobayashi*, Rie Nogita* and \\ Mutsumi Aoyagi ${ }^{* \dagger}$ \\ ${ }^{*}$ Research Institute for Information Technology, Kyushu University, Fukuoka 812-8581, Japan \\ ${ }^{\dagger}$ PSI Project Lab., Kyushu University, 3-8-33-710 Momochihama, Sawara-ku, Fukuoka 814-0001, Japan \\ ${ }^{* *}$ Fukuoka IST Foundation, Acros Fukuoka Nishi Office 9F, 1-1-1 Tenjin, Chuo-ku, Fukuoka 810-0001, Japan \\ ${ }^{\ddagger}$ Department of Informatics, Kyushu University, 6-10-1 Hakozaki, Higashi-ku, Fukuoka 812-8581, Japan
}

\begin{abstract}
OpenFMO framework, an open-source software (OSS) platform for Fragment Molecular Orbital (FMO) method, is extended to multi-physics simulations (MPS). After reviewing the several FMO implementations on distributed computer environments, the subsequent development planning corresponding to MPS is presented. It is discussed which should be selected as a scientific software, lightweight and reconfigurable form or large and self-contained form.
\end{abstract}

Keywords: fragment molecular orbital method, reference interaction site model, multi-physics simulation, open-source software PACS: 02.70.-c, 05.10.-a, 31.15.-p

\section{INTRODUCTION AND OVERVIEW}

Multi-physics simulations are widely used even in complex scientific studies. Such calculations are often constructed by combining multiple theories including different degrees of approximations and different scales of description. Since reality and accuracy are required increasingly, these simulations have become larger and more complicated year by year. Grids, distributed computer resources over wide-area networks, are expected to execute such complicated scientific applications, and have been installed all over the world in order to demonstrate large-scale heterogeneous simulations with the help of middlewares [1,2]. On the other hand, the next generation supercomputer with a petascale performance is already planned in several countries[3,4]. Thus, the development of high-performance computing environments is fast and transient. As a scientist, it is important to watch the trend of those computer resources.

In the present contribution, the multi-physics calculations by Fragment Molecular Orbital (FMO) method [5] are constructed on the distributed computing environments. OpenFMO framework toward "peta-scale" computing[6, 7] is extended to the multi-physics simulations. It is also discussed what architecture and development policy should be chosen in the fast-moving world of computing.

\section{GRID-ENABLED CALCULATIONS OF FMO}

Before entering the main subject, we briefly review the grid-enabled FMO implementations developed in the NAREGI project[1]. These are based on the famous MO package, GAMESS[8].

\section{Implementation of a Loosely-coupled FMO}

Although it is usually considered as an approximation to ab initio molecular orbital (MO) calculations, the FMO algorithm is a multi-layered problem (see Fig. 1(b)) including the MO calculations for each fragment and the electrostatic (ES) interaction between fragments. In the MO-layer, the quantum mechanical interactions of all the atoms and electrons within a fragment are included to obtain a fragment energy. On the other hand, only the classical ES interaction is considered when we go over the boundary of fragments. Since the MO-layer calculations can be executed independently, we can break the program into loosely-coupled components corresponding to a large-scale parallel execution in the distributed computing environments (Fig. 1(c)). 

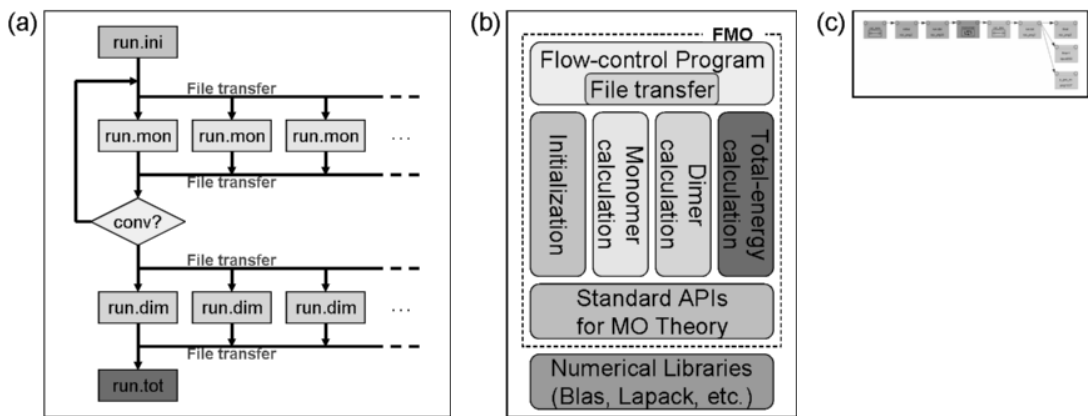

FIGURE 1. The structure of Loosely-coupled FMO represented in (a) a flow chart, (b) a program stack, and (c) graphical icons on the NAREGI Workflow tool.
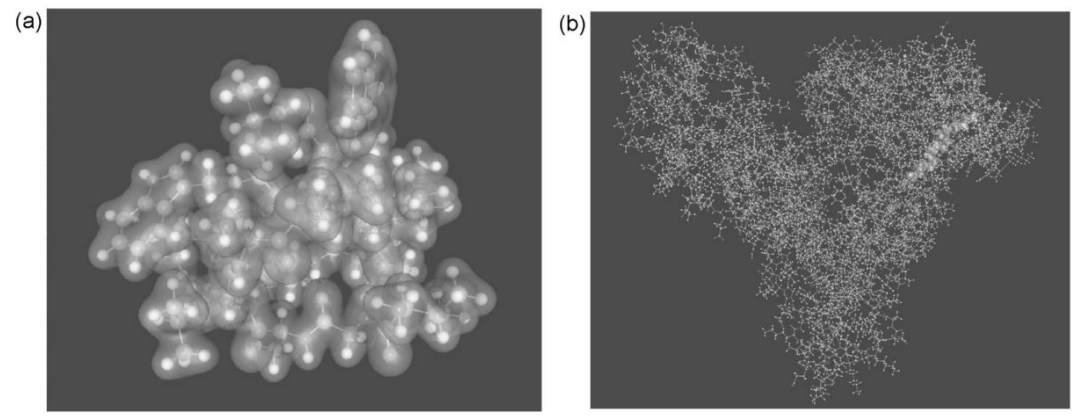

FIGURE 2. The electron density obtained by FMO is shown by the use of the NAREGI visualization system: (a) the total electron density of a Gramicidin-A (1JNO); (b) the electron density of a fragment in a fatty acid-albumin.

The grid-enabled version called "Loosely-coupled FMO" was developed as a part of NAREGI[1]. The total control flow is constructed by the use of the NAREGI Workflow tool. In Fig. 2 (a), the total electron density of the whole molecule[9] of a Gramicidin-A is shown as an equi-density surface, and the electron density for one of the fragments in a fatty-acid albumin is shown in Fig. 2(b).

\section{D-RISM/FMO Simulation Connected by a Mediator}

As an example of the multi-physics simulations, a coupled simulation of FMO and 3D-RISM is presented, where FMO calculations are coupled to statistical mechanics calculations for molecular liquids by Reference Interaction Site Model (RISM)[10]. In order to obtain properties of bio-molecules, drugs, enzymes, etc., it is necessary to perform calculations under the influence of a solvent since these molecules usually work in aqueous solution. However, the full description of the solute and solvent system is difficult in general because of the large number of degrees of freedom. The standard strategy to solve the problem is to combine, in some way, originally different theories or programs, which is the multi-physics approach.

In the multi-physics simulations, physical data are exchanged between separate program components, where we must transform not only formats but also their semantics, i.e., physical meanings of the data. In order to assist such dataexchanges with semantic transformations, we used a set of application program interfaces called Mediator (mediatorAPI) [11, 1], which is included in the beta-version release of the NAREGI grid-middleware. Fig. 3(a) shows the total flow of this simulation, where the partial charge distribution of the solute and solvent molecules are exchanged each other through the mediator-API (Fig. 3(b)). In order to execute on the NAREGI grid, the flow is incorporated in the NAREGI Workflow tool (Fig. 3(c)).

In Fig. 4, we show results of this coupled calculation for methionine-enkephalin (75 atoms) and chignolin (138 atoms) in aqueous solution, where the partial charge distribution by water molecules are also shown around these molecules. 

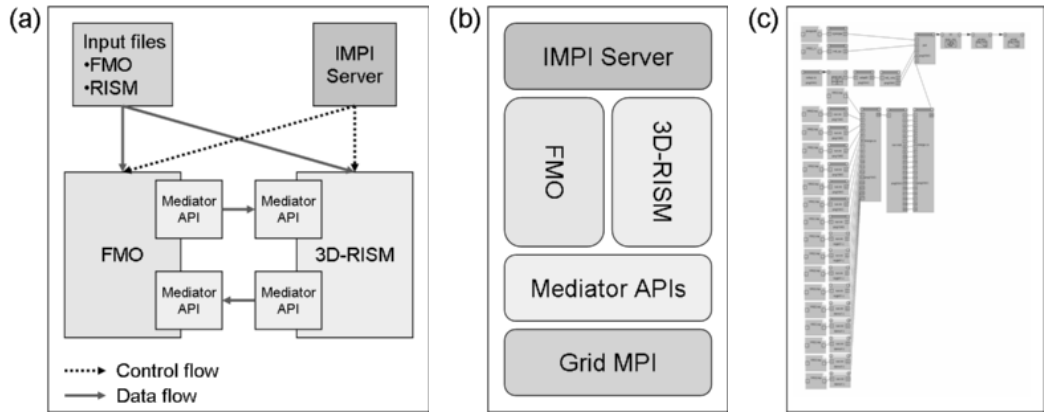

FIGURE 3. The structure of 3D-RISM/FMO represented in (a) a flow chart, (b) a program stack, and (c) graphical icons on the NAREGI Workflow tool.
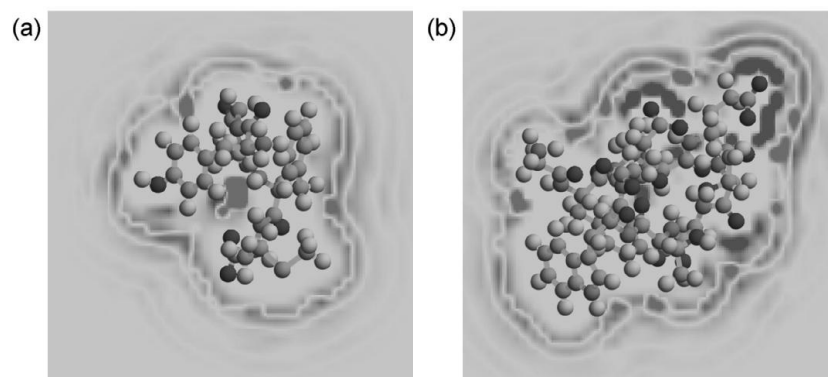

FIGURE 4. Charge distribution of water around a solute molecule obtained by 3D-RISM/FMO coupled simulations: (a) methionine-enkephalin (1PLW, 75 atoms); (b) chignolin (1UAO, 138 atoms).

\section{DEVELOPMENT OF OPENFMO FRAMEWORK}

OpenFMO[6] is an open-licensed software platform to construct FMO applications under high-performance distributed computer environments. The current status of this development is in the end of Phase II. In Phase I, we introduced the OpenFMO framework and predicted a peta-scale performance on a hypothetical computer architecture[7]. In Phase II, we have tried to implement the skeleton by one-sided communications[12] under the PSI project[4]. In Phase III, we are going to extend the platform to the multi-physics simulations (see Fig. 5).

\section{Multi-physics Extension of OpenFMO and its Application}

The main purpose of the Phase II in the development schedule of OpenFMO (Left of Fig. 5) was to correspond actual executions on the next generation supercomputer with more than 10,000 CPUs, where we have tried to reduce redundant memory consumption on each computing nodes and improve parallel performance by the use of one-sided communications. The detailed results will be presented elsewhere[12].

The OpenFMO framework is extended to correspond to multi-physics applications including scientific simulations (Fig. 5). Since the current FMO skeleton is well configured and has been proven effective in high-performance computing environments, it is better that the other multi-physics components are developed separately. Then, the key point is physical data representation and manipulation, where sort of transparent accessibility to internal data from outer components should be provided. The semantic transformation of the data can also be executed by the Mediator component depending on the needs.

By the use of the multi-physics extension, one can construct the following coupled simulations: QM/MM or QM/MD calculations $[13,14]$ for docking simulations of proteins and enzymes in aqueous solution, RISM/SCF simulations[10] for various protein molecules with scientific theoretical studies[15], etc. One of the properties of the OpenFMO platform is the lightweight and reconfigurable skeleton program, which is useful for modifying applications corresponding to fast-changing computational environments. 
Phase I (2006)

- Fixing interfaces of MO library programs

- Skeleton with GAMESS-type algorithm

- Performance prediction for peta-computing

Phase II (the first half of 2007)

$\circ$ New skeleton for high-performance computing

- Performance analysis using MPI-2 functions

Phase III (the latter half of 2007)

- Multi-physics extension

- Scientific applications

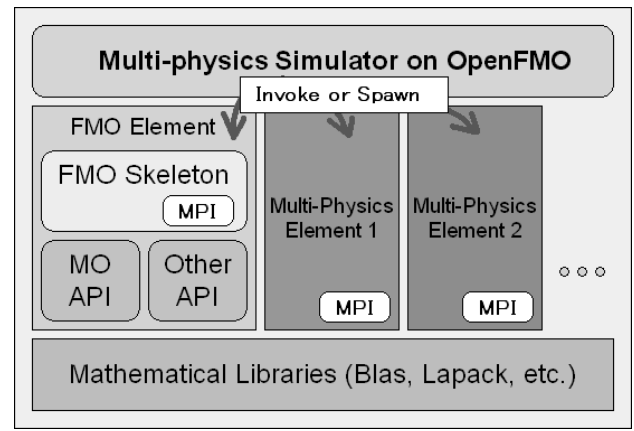

FIGURE 5. Left: Development planning of OpenFMO. Right: Stack structure of OpenFMO with multi-physics extension.

\section{DISCUSSION}

Application sizes of recent molecular package programs are more and more increasing by their self-contained structures with many functions corresponding to complicated options and various computer environments. Such strategies in their development potentially run into a dead-end. However, when we use multiple grid resources simultaneously, it is inevitable to prepare a tailored scheduler[16] in each application. The most important task as a scientist is to develop effective theories and algorithms that can be implemented on every computer environments, while actual implementation on a given environment should be carefully done with the help of computer scientists. Our development of the OpenFMO framework is one of those attempts to implement the FMO algorithm on the future computers.

This work is partly supported by the Ministry of Education, Sports, Culture, Science and Technology (MEXT) through the Science-grid NAREGI Program under the Development and Application of Advanced High-performance Supercomputer Project.

\section{REFERENCES}

1. S. Matsuoka, S. Shimojo, M. Aoyagi, S. Sekiguchi, H. Usami, and K. Miura, "Japanese computational grid research project: NAREGI," in Proceedings of IEEE, 2005, vol. 93, p. 522.

2. NAREGI Project, URL http: //www. naregi.org/.

3. Riken Supercomputer Project, URL http://www.nsc.riken.jp/.

4. PSI project, URL http://www . psi-project.jp/.

5. K. Kitaura, E. Ikeo, T. Asada, T. Nakano, and M. Uebayasi, Chem. Phys. Lett. 313, 701-706 (1999).

6. OpenFMO, URL http: / / www . openfmo.org/OpenFMO.

7. T. Takami, J. Maki, J. Ooba, Y. Inadomi, H. Honda, T. Kobayashi, R. Nogita, and M. Aoyagi, "Open-architecture Implementation of Fragment Molecular Orbital Method for Peta-scale Computing," to appear in Proceedings of the 2nd IEEE/ACM international workshop on high performance computing for nano-science and technology (HPCNano06), 2007, CS.DC/ 0701075.

8. GAMESS, URL http://www.msg.ameslab.gov/GAMESS/GAMESS.html.

9. Y. Inadomi, T. Nakano, K. Kitaura, and U. Nagashima, Chem. Phys. Lett. 364, 139 (2002).

10. F. Hirata, editor, Molecular Theory of Solvation, Kluwer Academic Pub., 2003.

11. S. Ho, S. Itoh, S. Ihara, and R. D. Schlichting, "Agent middleware for heterogeneous scientific simulations," in Proceedings of ACM/IEEE SC 1998 Conference (SC'98), 1998, p. 15.

12. J. Maki, Y. Inadomi, T. Takami, R. Susukita, H. Honda, J. Ooba, T. Kobayashi, R. Nogita, K. Inoue, and M. Aoyagi, "One-sided Communication Implementation in FMO Method," to appear in Proceedings of the 9th international conference in high performance computing in Asia Pacific region (HPCAsia07), 2007.

13. M. J. Field, P. A. Bash, and M. Karplus, J. Comput. Chem. 11, 700-733 (1990).

14. Y. Komeiji, T. Nakano, K. Fukuzawa, Y. Ueno, Y. Inadomi, T. Nemoto, M. Uebayasi, D. G. Fedorov, and K. Kitaura, Chem. Phys. Lett. 372, 342-347 (2003).

15. T. Takami, J. Maki, J. Ooba, T. Kobayashi, R. Nogita, and M. Aoyagi, J. Phys. Soc. Jpn. 76, 013001 (2007), nlin.CD/0611042.

16. T. Ikegami, J. Maki, T. Takami, Y. Tanaka, M. Yokokawa, S. Sekiguchi, and M. Aoyagi, "GridFMO - Quantum Chemistry of Proteins on the Grid," to appear in Proceedings of the 8th IEEE/ACM International Conference on Grid Computing (Grid 2007), 2007. 